\title{
A global look at local translation
}

Proximity-specific ribosome profiling reveals the exquisite specificity of translation at the endoplasmic reticulum and mitochondrial outer membrane.

Subcellular compartmentalization is crucial for the proper function of an organism. One way cells compartmentalize protein function is by synthesizing them when and where they are needed. However, local translation has proven difficult to study. According to Jonathan Weissman at the University of California at San Francisco and the Howard Hughes Medical Institute, methods for studying local translation on the proteomic scale disrupt the cell and are applicable only to some subcellular locations.

To study local translation in intact cells, the Weissman lab's Calvin Jan, a postdoctoral fellow, and Christopher Williams, a graduate student, developed proximity-specific ribosome profiling, which extends the ribosomeprofiling method previously developed in the Weissman lab to study a subset of translating ribosomes. During ribosome profiling, mRNA fragments protected by ribosomes undergoing translation are analyzed by deep sequencing, providing a snapshot of translation in a cell.

In proximity-specific ribosome profiling, the biotin ligase BirA is expressed as a fusion to a protein that localizes to a specific compartment. This BirA fusion is coexpressed with ribosomes that contain a biotin acceptor peptide. Ribosomes that come in close contact with the biotin ligase are thus biotinylated. This subset of ribosomes is then subjected to streptavidin pulldown and ribosome profiling.

Although the method is an extension of an existing approach, Weissman notes that its development was not without its challenges, particularly for controlling the timing of biotinylation. This required finding conditions in which the cells were growing happily but biotin levels were too low to label ribosomes, appropriately pulsing in biotin, and effectively quenching biotinylation after cell lysis.

The team studied local translation in the endoplasmic reticulum (ER) and the mitochondrial outer membrane. The method allowed them to shed light on many questions regarding cotranslational translocation of membrane proteins.

First, the researchers found that most secretory proteins are targeted to the ER cotranslationally, which was surprising because signal-recognition particle (SRP)independent proteins are also known to be capable of translocating post-translationally in vitro (Jan et al., 2014). Moreover, they found that targeting relies more on the length of the coding region after the ER-targeting signal than on dependence on SRP.

The team also found that the translocon component Sec66 is responsible for translocation of a subset of SRP-independent proteins. Whereas most proteins are recognized for ER targeting once the signal sequence emerges from the ribosome, this class of proteins contains signal sequences that require $\sim 120$ amino acids to be targeted. This longer nascent chain may adopt an alternate topology necessary for translocation. According to Weissman, these results "show us an example where there really is a true functional diversity of signal sequences."

The researchers were also able to address whether mitochondrial proteins are cotranslationally translocated (Williams et al., 2014). Though many of the proteins tested are capable of being imported into mitochondria post-translationally, Weissman's team revealed that $\sim 30 \%$ of mitochondrial reference genes are cotranslationally targeted.

The researchers wondered whether proteins could be targeted to both the ER and mitochondria. Although the vast majority are targeted to only a single organelle, they observed one prominent exception, Osm1, which is targeted to both. By analyzing their data, they found that Osm 1 has two translational start sites, which produce two forms of the protein with distinct targeting signals. These results demonstrate how precisely cells can regulate the compartmentalization of proteins.

As a whole, this work highlights the power of proximity-specific ribosome profiling in uncovering the secrets of local translation on the global scale. Future use of this technique should greatly enhance our understanding of how cells such as neurons compartmentalize reactions to ensure proper protein function. Rita Strack

\section{RESEARCH PAPERS}

Jan, C.H. et al. Principles of ER cotranslational translocation revealed by proximity-specific ribosome profiling. Science 346, 1257521 (2014).

Williams, C.C. et al. Targeting and plasticity of mitochondrial proteins revealed by proximity-specific ribosome profiling. Science 346, 748-751 (2014). 